\title{
Mechanical spectroscopy of laser deposited polymers
}

\author{
Andreas Meschede • Thorsten Scharf • \\ Hans-Ulrich Krebs • Konrad Samwer
}

Received: 12 October 2007 / Accepted: 4 March 2008 / Published online: 16 June 2008

(C) The Author(s) 2008. This article is published with open access at Springerlink.com

\begin{abstract}
Pulsed laser deposition (PLD) at $248 \mathrm{~nm}$ in ultra high vacuum was used to produce thin poly(methyl methacrylate) (PMMA) and poly(ethyl methacrylate) (PEMA) films. The ablation and deposition mechanisms were found to be similar in both systems. Having the same backbone, these polymers differ in the size of their polar side groups leading to changes in their dynamics. Studies of the relaxation processes were performed using mechanical torsion and bending spectroscopy by means of a doublepaddle oscillator (DPO) and an in-situ plasma plume excited reed (PPXR), respectively. A strong increase of the mechanical damping was observed during annealing of the polymer films well above the glass transition temperature $T_{\mathrm{g}}$, while in-situ X-ray measurements did not reveal any structural changes. For PEMA, the glass transition temperature $T_{\mathrm{g}}=335 \mathrm{~K}$ and the main absorption maximum appear at lower temperatures compared to PMMA $\left(T_{\mathrm{g}}=380 \mathrm{~K}\right)$, allowing one to measure the mechanical properties in a much wider range above $T_{\mathrm{g}}$.
\end{abstract}

PACS 68.60.Bs $\cdot$ 81.05.Lg $\cdot 62.40 .+\mathrm{i} \cdot 83.85 . \mathrm{Vb} \cdot 82.35 . \mathrm{Lr}$

\section{Introduction}

Recently, pulsed laser deposition (PLD) has been shown to be a flexible method to produce high quality polymer thin

\footnotetext{
A. Meschede ( $\varangle) \cdot$ T. Scharf · H.-U. Krebs

Institut für Materialphysik, University of Göttingen,

Friedrich-Hund-Platz 1, 37077 Göttingen, Germany

e-mail: meschede@ump.gwdg.de

K. Samwer

I. Physikalisches Institut, University of Göttingen,

Friedrich-Hund-Platz 1, 37077 Göttingen, Germany
}

films. Especially for poly(methyl methacrylate) (PMMA), the ablation and deposition process is still a matter of discussion [1-4]. But using high laser fluences in the range of $1 \mathrm{~J} / \mathrm{cm}^{2}$ at a wavelength of $248 \mathrm{~nm}$ the main mechanism could be identified as an incubation process [5] followed by transport of macromolecules mainly via droplet transport [6]. Consequently, in this paper we compare the results obtained for PMMA with those of laser deposited poly(ethyl methacrylate) PEMA. These polymers consist of an identical backbone and only differ in the size of their polar side group. The structural formulae are depicted in the inset of Fig. 3.

Thinking of any commercial application for thin polymer films, as coatings, resists, or even biocompatible buffers, their mechanical properties are of special interest. Dynamics in complex amorphous polymeric systems are characterised by a variety of different inner vibrations. For PMMA the processes dominating the glass transition were found to be movements of whole chains and chain segments, mainly tilting and rotational vibrations ( $\alpha$-relaxation) [7], and additionally flipping movements of the whole ester side group ( $\beta$-relaxation) [8]. Depending on the frequency and temperature at which the system is probed, both processes may overlap (merging [9]). Comparing dielectric and mechanical spectroscopy measurements for laser deposited PMMA, the mechanical damping maximum could be identified as dominated by $\alpha$-relaxation within the polymer [10].

In this paper we shall study the influence of the elongated side group in PEMA on its microscopic relaxation and dynamics, focusing on potential additional relaxation modes and $\alpha-\beta$ merging effects by using two complementary spectroscopy methods, the ex-situ and highly sensitive double paddle oscillator (DPO) [11] and the in-situ plasma plume excited reed (PPXR) [12] methods. 


\section{Experimental setup}

PMMA and PEMA films were deposited on polycrystalline $\mathrm{Al}$, single crystalline $\mathrm{Si}(111)$ substrates, DPOs and vibrating reeds, respectively, at room temperature. $\mathrm{A} \mathrm{KrF}$ excimer laser with a wavelength of $248 \mathrm{~nm}$, pulse duration of $30 \mathrm{~ns}$ at a repetition rate of $10 \mathrm{~Hz}$ was used. The polymer films were deposited at a laser fluence of $1.2 \mathrm{~J} / \mathrm{cm}^{2}$ in an ultra-high vacuum chamber with a base pressure of about $10^{-8} \mathrm{mbar}$. The target-to-substrate distance was $50 \mathrm{~mm}$. The setup has been described in more detail before [13]. As polymer targets, hot-pressed powders (Aldrich, $10^{6} \mathrm{~g} / \mathrm{mol}, 120^{\circ} \mathrm{C}$ at $6 \times 10^{7} \mathrm{~N} / \mathrm{m}^{2}$ ) were used. The microstructure of the polymer films was investigated by scanning electron microscopy (SEM, Cambridge Instruments S360). Fourier-transform infrared (FT-IR) spectra of the films deposited on $\mathrm{Al}$ substrates were taken in reflection mode on a Bruker Tensor 37. Insitu X-ray diffraction (XRD) patterns were recorded up to temperatures of $440 \mathrm{~K}$ using a Siemens D500 diffractometer with $\mathrm{Co}-\mathrm{K}_{\alpha}$ radiation.

Mechanical spectroscopy was performed with two different setups. For high resolution measurements, necessary to study relaxation processes during the annealing steps of the polymers, double paddle oscillators $[10,11]$ were used as substrates. A polymer film about $1 \mu \mathrm{m}$ thick was deposited on the DPO and the mechanical loss was ex-situ investigated at temperatures up to $575 \mathrm{~K}$ at a torsion vibration mode with a resonance frequency of about $5.4 \mathrm{kHz}$. A conventional electrostatic setup is used for driving the paddle and detecting its amplitude. Second, an in-situ technique was developed (see Fig. 1) consisting of a vibrating reed $(1 \mathrm{~mm} \times 8-30 \mathrm{~mm} \times 120 \mu \mathrm{m} \mathrm{Si}(100)$ single crystal $)$, which was mounted in the PLD chamber on a (liquid nitrogen) cooling stage with a resistance heater allowing one to measure at temperatures of between $200 \mathrm{~K}$ and $600 \mathrm{~K}$. After deposition of the polymer film, for each single measurement the Si substrate was excited by the momentum of the expanding plasma plume coming from an Ag target illuminated by a laser pulse of about $3 \mathrm{~J} / \mathrm{cm}^{2}$. Driven by the impact

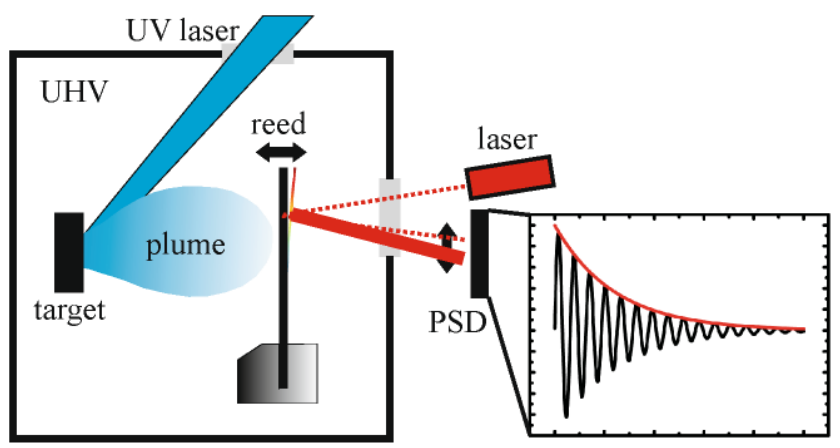

Fig. 1 Sketch of the PPXR principle. A vibrating reed is excited by the momentum of the Ag plasma plume, and the damped oscillations are optically recorded of the incident material pulse, the reed is instantaneously pushed to one side and afterwards performs a damped vibration mainly in its first bending mode between 500 and $8000 \mathrm{~Hz}$, depending on the reed geometry. The oscillations of the reed and their damping are measured using the reflections of a diode laser beam from the backside of the substrate on a position sensitive detector. More details of the PPXR setup are described in [12]. Both methods have in common that the damping vs. temperature of the bare substrates has to be measured before deposition of the polymer, and this has to be subtracted from the raw data.

\section{Results and discussion}

First we compare the microstructure and the structural properties of the PEMA films after deposition at room temperature with those of PMMA films. As described earlier [6, 14], PMMA films deposited at $248 \mathrm{~nm}$ at high laser fluences (above $1 \mathrm{~J} / \mathrm{cm}^{2}$ ) mainly consist of a large amount of droplets $(\sim 80 \mathrm{wt} \%)$ with an average molecular mass of $8.000 \mathrm{~g} / \mathrm{mol}$, expelled from the target due to strong subsurface heating in this low absorbing polymer, in agreement with the results of Blanchet [1]. Additionally a very smooth, highly cross-linked and non-soluble thin film is formed partly covering these droplets. It should be mentioned here that the formation of droplets can be avoided by using laser fluences close to the deposition threshold $\left(\sim 60 \mathrm{~mJ} / \mathrm{cm}^{2}\right)$ [15], leaving the main film properties comparable to those of the films deposited at higher energy densities.

As can be seen in Fig. 2, also the surface morphology of a PEMA film shows a high number of droplets. The microstructure is very similar to that of the PMMA films. In Fig. 3, the FT-IR spectrum of a laser deposited PEMA film is compared to the spectrum of PMMA. As expected, the

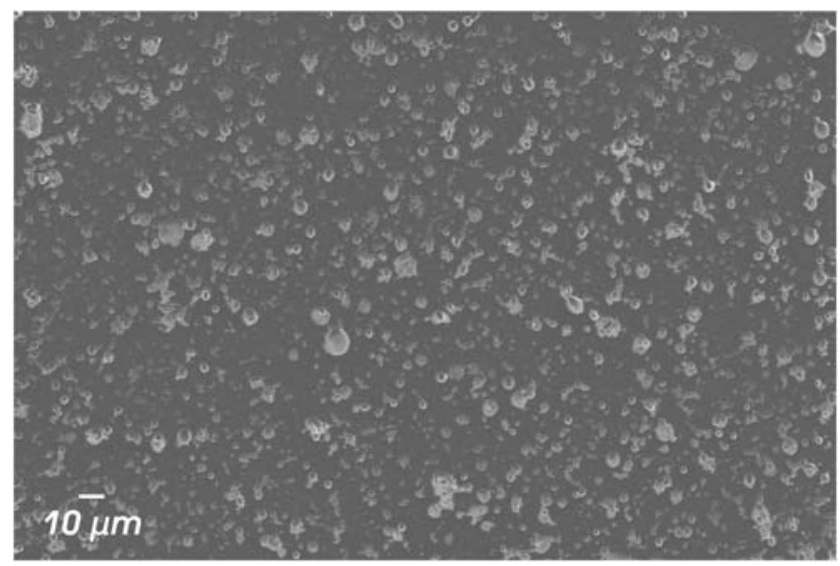

Fig. 2 Scanning electron micrograph of a poly(ethyl methacrylate) thin film prepared by 300 laser pulses at a laser energy density of $1.2 \mathrm{~J} / \mathrm{cm}^{2}$. The average film thickness is about $200 \mathrm{~nm}$ 


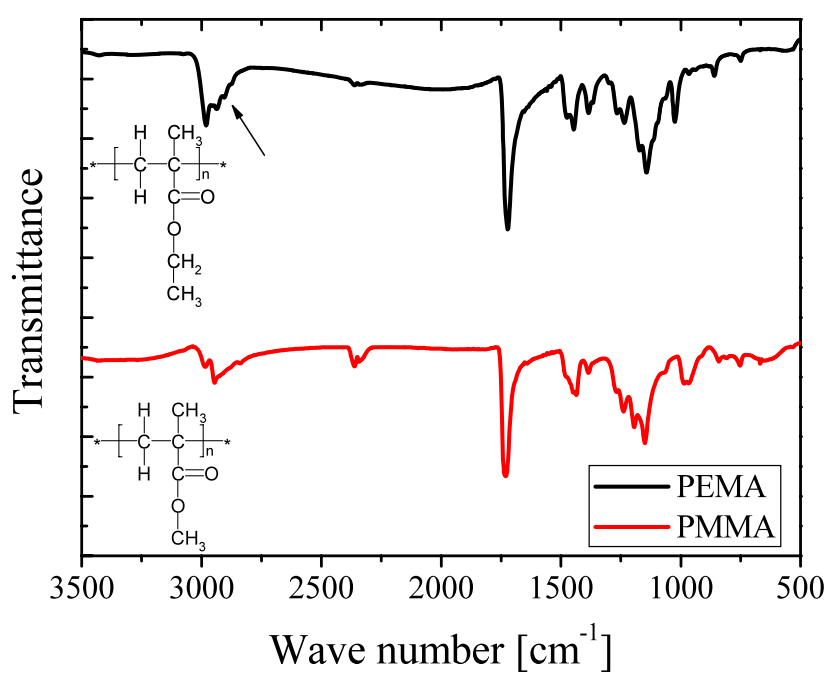

Fig. 3 Comparison of the FT-IR spectra of PMMA and PEMA thin films. The main change in the absorption bands due to the additional $\mathrm{CH}_{2}$ vibrational mode in the side chain is marked by an arrow

functional groups of the two polymers are almost the same, clearly indicating the relationship of both materials. Nevertheless, differences between the spectra occur due to the additional $\mathrm{CH}_{2}$ group. This can be seen especially at wave numbers around $2900-3000 \mathrm{~cm}^{-1}$, where the symmetric and asymmetric $\mathrm{CH}_{2}$ and $\mathrm{CH}_{3}$ stretch vibrations are observed. Here an additional absorption band (marked in Fig. 3 by an arrow) is observed in the case of PEMA.

To study relaxation processes inside the PEMA films, mechanical torsion loss spectra were taken by DPO measurements during gradual annealing of the films up to high temperatures. The high accuracy of this technique allows for detecting small changes in the loss spectra during relaxation (see Fig. 4). The plotted quantity, $\Delta\left(Q^{-1}\right)$, is the difference of the inverse damping factor $Q^{-1}$ :

$Q^{-1}=-\frac{\Lambda}{\pi f_{0}}$

with the logarithmic decrement $\Lambda$ and the resonance frequency $f_{0}$ for the system substrate/film and that for the bare substrate. For the films discussed here, the main contribution to the damping originates from the droplet component. As can be seen, dramatic relaxation effects occur in the mechanical loss spectra, when an as-deposited PEMA film is successively heated to higher temperatures. In this experiment the sample was heated to $425 \mathrm{~K}$ with a heating rate of $0.3 \mathrm{~K} / \mathrm{min}$, cooled down to room temperature, then heated to $465 \mathrm{~K}$ and cooled down again, and so on. The glass transition temperature $T_{\mathrm{g}}$ of the polymer film can be determined, as the onset of the energy absorption increases, to be about $335 \mathrm{~K}$, which is in agreement with the value of 330-340 K observed for bulk material. Above $T_{\mathrm{g}}$, viscous flow sets in

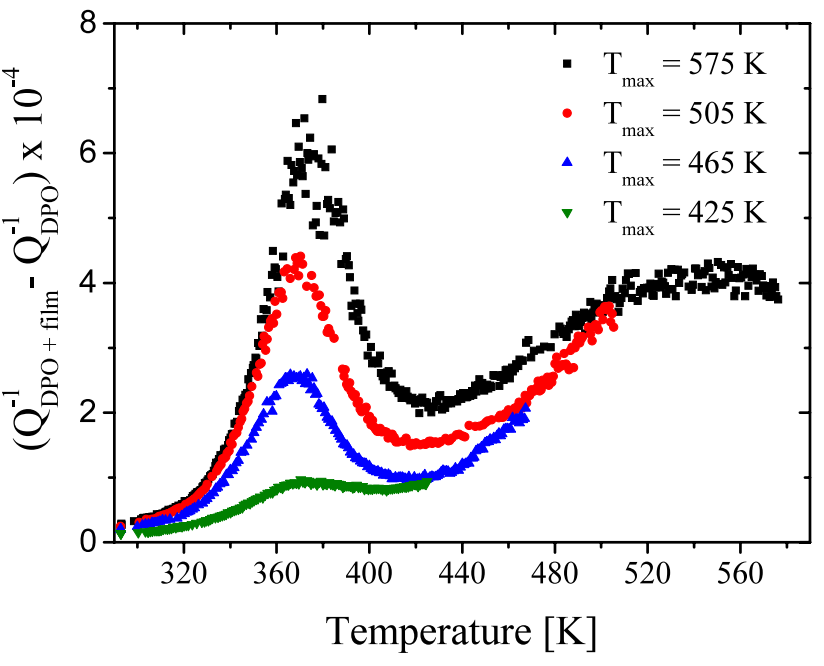

Fig. 4 Changes of the mechanical damping coefficient of an about $1 \mu \mathrm{m}$ thick PEMA film during subsequent heating temperature cycles due to an increasing amount of relaxation

at $365 \mathrm{~K}$ and a strong increase of the maximum loss factor is observed with increasing final heating temperature. The shape of the observed peak is very similar to those for PMMA films [10], not showing any additional relaxation modes. Thus we conclude that despite the elongated side group, also in PEMA the $\alpha$-processes are dominating the relaxation behaviours.

With elevated annealing temperature not only the maximum damping mainly in the $\alpha$-peak region is strongly increased, but the absorption also increases at all temperatures lower than the former highest temperature. This indicates that at the highest temperatures new (slower) damping processes arise corresponding to movements of larger chain segments. Vibrational modes inside the polymer frozen in during the deposition process are released and now participate in the frictional movements inside the polymer. The actuation of these slow vibrational modes is accompanied by a release of vibrations of smaller segments, inducing an overall increment of damping up to the highest temperature reached.

The increase of the damping above $430 \mathrm{~K}$ probably belongs to handicapped slipping motions of polymer chains within the molten state, which are connected with strong friction. Above $540 \mathrm{~K}$, the absorption curve decreases again due to a partial evaporation of the polymer.

For PMMA it is known from SEM investigations that annealing does not lead to morphological changes. Furthermore size exclusion chromatography (SEC) measurements revealed that subsequent annealing of PMMA films does not affect the average molecular weight, although the deposition at elevated substrate temperatures leads to an increase of molecular mass [6]. To investigate whether the relaxation effects are associated with structural changes, the PEMA thin 


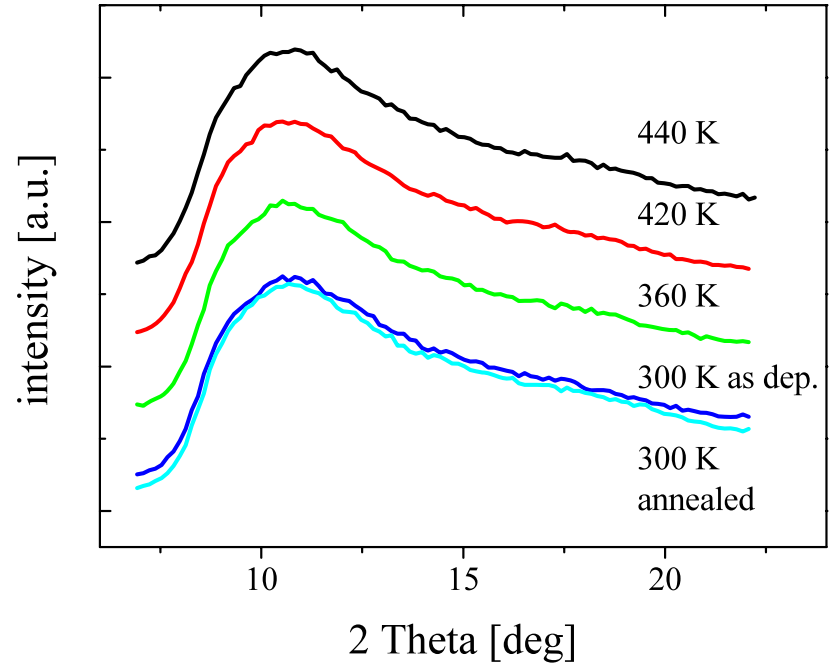

Fig. 5 Stacked X-ray diffraction patterns during heating of PEMA at high temperatures, showing almost no structural changes during relaxation

films were studied during annealing up to $440 \mathrm{~K}$ by in-situ $\mathrm{X}$-ray diffraction (Fig. 5). The room temperature measurement shows a main amorphous halo at about $2 \theta=11^{\circ}$ (corresponding to a distance of $0.7 \mathrm{~nm}$ ) with a slight shoulder at about $19^{\circ}(1.2 \mathrm{~nm})$. Clearly distinguishable maxima as e.g. seen by Wind et al. [16] are not found, this being a sign for lack of nanophase separation in this pulsed laser deposited material. The spectra show no significant changes during heating well above $T_{\mathrm{g}}$. Even after cooling down to room temperature again only marginal differences in the patterns appear when directly compared with that after deposition. Therefore it can be concluded that the relaxation is not connected with significant structural changes or a strong healing out of free volume distributed within the sample.

Figure 6 shows the comparison of the temperaturedependent mechanical loss curves for both materials, PEMA and PMMA, obtained by PPXR measurements at 1750 and $2500 \mathrm{~Hz}$, respectively. For these experiments, the samples were heated to a temperature slightly above the main damping peak before the measurement to ensure a widely relaxed state inside the polymer. In both cases, the bending loss spectra are dominated by a broad maximum and a similar shape of the damping peaks. Compared to the results of PMMA, the damping maximum of PEMA is shifted to lower temperatures due to a lower glass transition temperature, but no new relaxation modes are found in this temperature regime. The changes to lower temperatures can be attributed to the increase in the length of the side group and therefore the insertion of free volume in the polymer, which weakens the PEMA in comparison to PMMA.

When comparing the DPO (Fig. 4) with the PPXR measurements (Fig. 6) the differences between the damping spectra indicate that the latter method is more sensitive for

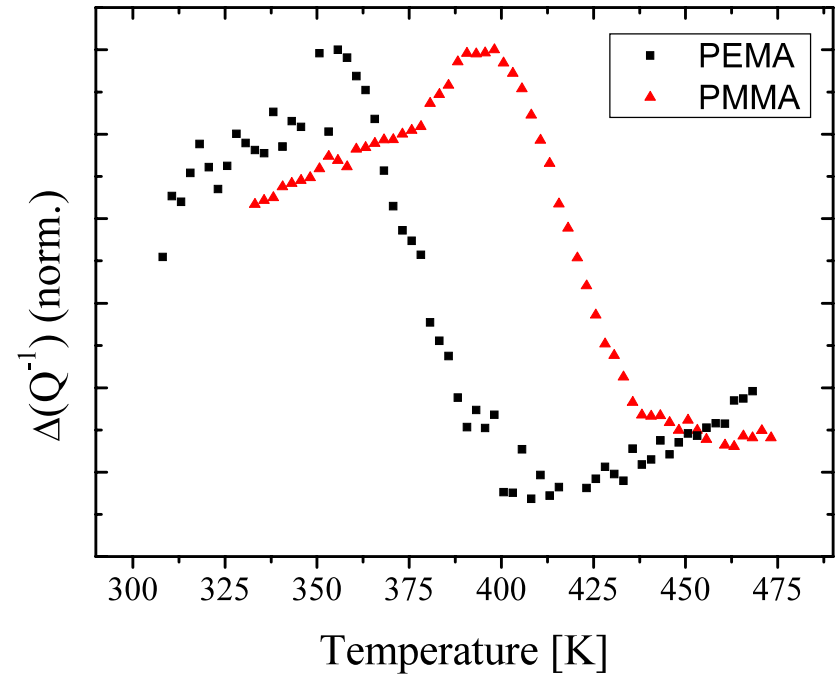

Fig. 6 Normalized inverse quality factor of PMMA and PEMA thin films obtained by PPXR. The decreased glass transition temperature can easily be seen by the shift in the temperature of the loss maximum

faster processes below $T_{\mathrm{g}}$, as here a low temperature flank is observed. These differences are probably caused by the intrinsic differences of the main motions of the two methods, but they cannot be explained by the lower working frequencies of the PPXR. The DPO motion is dominated by a torsion vibration receptive on the shear modulus, while the PPXR's vibrating reed mainly performs a bending motion representing the elastic modulus.

\section{Conclusion}

In summary, we have shown that although the deposition mechanisms, microstructure and composition of laser deposited PEMA and PMMA films are similar, their macroscopic mechanical properties strongly differ while their inner dynamics show no significant changes. The largest obvious difference between PEMA and PMMA, induced by the elongation of the side chain, is a softening of the PEMA polymer, visible in a shift of the glass transition temperature and the maximum loss to lower temperatures. In both polymers, with increasing annealing temperature cumulative relaxation occurs, induced by a healing out of larger sections of the polymer chains increasing the amount of all length scales participating in the frictional movement. While relaxation effects (and very thin films) can be investigated using the ex-situ DPO technique, PPXR allows one to measure the temperature dependence of the mechanical loss without breaking the vacuum. The combination of the high accuracy of the DPO measurement and the in-situ capability of the PPXR technique within the PLD chamber is under way.

Acknowledgement This work was supported by the Deutsche Forschungsgemeinschaft (SFB 602 and GK 782). 
Open Access This article is distributed under the terms of the Creative Commons Attribution Noncommercial License which permits any noncommercial use, distribution, and reproduction in any medium, provided the original author(s) and source are credited.

\section{References}

1. G.B. Blanchet, Macromolecules 28, 4603 (1995)

2. D.B. Chrisey, A. Piqué, R.A. McGill, J.S. Horwitz, B.R. Ringeisen, D.M. Bubb, P.K. Wu, Chem. Rev. 103, 553 (2003)

3. P.E. Dyer, Appl. Phys. A 77, 167 (2003)

4. S.G. Hansen, T.E. Robitaille, Appl. Phys. Lett. 52, 81 (1988)

5. S. Küper, M. Stuke, Appl. Phys. A 49, 211 (1989)

6. E. Süske, T. Scharf, H.U. Krebs, T. Junkers, M. Buback, J. Appl. Phys. 100, 014906 (2006)

7. S.C. Kuebler, D.J. Schaefer, C. Boeffel, U. Pawelzik, H.W. Spiess, Macromolecules 30, 6597 (1997)
8. K. Schmidt-Rohr, A.S. Kulik, H.W. Beckham, A. Ohlemacher, U. Pawelzik, C. Boeffel, H.W. Spiess, Macromolecules 27, 4733 (1994)

9. R. Bergman, F. Alvarez, A. Alegría, J. Colmenero, J. Chem. Phys. 109, 7546 (1998)

10. P. Rösner, J. Hachenberg, K. Samwer, R. Wehn, P. Lunkenheimer, A. Loidl, E. Süske, T. Scharf, H.U. Krebs, New J. Phys. 8, 89 (2006)

11. P. Rösner, K. Samwer, R.O. Pohl, S. Schneider, Rev. Sci. Instrum. 74, 3395 (2003)

12. T. Scharf, H.U. Krebs, Rev. Sci. Instrum. 77, 093901 (2006)

13. H.U. Krebs, Int. J. Non-Equilib. Process. 10, 3 (1997)

14. E. Süske, T. Scharf, H.U. Krebs, E. Panchenlo, T. Junkers, M. Egorov, M. Buback, H. Kijewski, J. Appl. Phys. 97, 063501 (2005)

15. B. Lösekrug, A. Meschede, H.U. Krebs, Appl. Surf. Sci. 254, 1312 (2007)

16. M. Wind, R. Graf, S. Renker, H.W. Spiess, W. Steffen, J. Chem. Phys. 122, 014906 (2005) 